\title{
Rebuttal comments on "A potential biomarker for objective diagnosis of overactive bladder: urinary nerve growth factor"
}

\author{
Bo Hai
}

Received: 18 November 2014 / Accepted: 18 November 2014 / Published online: 23 December 2014

(C) Springer Science+Business Media Dordrecht 2014

\section{Editor,}

We have read the letter from Mehmet Agilli et al. In "Materials and methods" section, as urge incontinence symptom is one of the OAB Symptom Score (OABSS) criteria, "no urge incontinence" is our writing error, the patients including urge incontinence, thank you for pointing out, and OABSS $12-14$ score class certainly contains urge incontinence symptom, as OAB-wet.

About exclusion criteria, they point out neuropsychiatric diseases, eating disorders, cardiometabolic diseases, metabolic syndrome, type 2 diabetes mellitus, obesity (BMIs), vitamin $\mathrm{D}$ analogues, zinc, vitamin $\mathrm{B} 12$, vitamin $\mathrm{A}$, and herbal medicines, and some drugs such as estrogen, glucocorticoids, acetyl-L-carnitine, and antipsychotics can affect Neurotrophic Factor expression. We think there is no need to exclude so many factors, according to the Reference papers, most of them affecting Neurotrophic Factor expression of other tissue, such as brain, fat, and circulating, not urine. We just excluded bladder tumor, bladder stones, interstitial cystitis, tuberculosis of bladder, etc., which may affect NF of urine. This is also standard practice for others [1-3].

\section{References}

1. Antunes-Lopes T, Pinto R, Carvalho-Barros S (2011) Urinary levels of Brain Derived Neurotrophic Factor (BDNF) in women with overactive bladder $(\mathrm{OAB})$ syndrome correlate with the severity of symptoms. Eur Urol Suppl 10(2):277-278

2. Liu HT, Kuo HC (2008) Urinary nerve growth factor level could be a potential biomarker for diagnosis of overactive bladder. $\mathbf{J}$ Urol 179(6):2270-2274

3. Kim JC, Park EY, Hong SH, Seo SI, Park YH et al (2005) Changes of urinary nerve growth factor and prostaglandins in male patients with overactive bladder symptom. Int $\mathrm{J}$ Urol 12(10):875-880

This comment refers to the article available at doi:10.1007/ s11255-014-0879-7.

B. Hai $(\bowtie)$

Wuhan, China

e-mail: bohai234@yeah.net 